Tema: Aciaria Elétrica

\title{
RECUPERAÇÃO DE ELEMENTOS DE LIGA DE RESÍDUOS SIDERÚRGICOS UTILIZANDO PELOTAS AUTORREDUTORAS*
}

\author{
Mário César Mantovani ${ }^{1}$ \\ Luiz Roberto Moraes Júnior ${ }^{2}$ \\ Luis Fernando Rosa ${ }^{3}$ \\ Elsomar Biancardi Guimaraes de Oliveira ${ }^{4}$ \\ Robson Leandro Silva ${ }^{5}$ \\ Egberto Antonio Possente ${ }^{6}$ \\ Almir Murari ${ }^{7}$ \\ Bruno Pessoa Ramos ${ }^{8}$ \\ Rafael Agnelli Mesquita ${ }^{9}$
}

\section{Resumo}

Um dos principais problemas enfrentados pelas aciarias é o descarte dos resíduos gerados durante a produção do aço. Além de se pagar pelo descarte em aterros monitorados, ocorre também a perda de elementos de liga, tais como $\mathrm{Cr}$, Ni, Mo, $\mathrm{V}$, etc. O objetivo deste trabalho é estudar a viabilidade de se aglomerar alguns resíduos gerados durante a produção do aço (lama de VOD/VD, poeira de forno panela e carepa de forjaria) na forma de pelotas autorredutoras e adiciona-las ao forno elétrico a arco (FEA). Primeiramente os resíduos foram misturados com finos de coque de petróleo e cimento. Após a pelotização e o período de cura, as pelotas autorredutoras foram fundidas num forno de indução (teste laboratorial), visando avaliar o nível de recuperação dos elementos de liga a partir dos resíduos. O rendimento dos elementos $\mathrm{Cr}$, Ni e Mo variaram de $84 \%$ a $99 \%$ nos teste laboratoriais. Nos testes industriais envolvendo as pelotas autorredutoras de carepa de forjaria (aço ferramenta H13), os rendimentos dos elementos $\mathrm{Cr}$, Mo e $\mathrm{V}$ variaram de 51\%-92\%, 94\%-99\% e 27,5\%-88\%, respectivamente.

Palavras-chave: Reciclagem; Resíduo; Pelotas.

\section{RECOVERY OF VALUABLE ELEMENTS FROM STEEL-MILL WASTES THROUGH SELF- REDUCING PELLETS}

\begin{abstract}
One of the most important problems encountered in minimill steel plants throughout the world is the disposition of wastes generated during the production of steel. Besides the payment for disposal in a controlled landfill site, many important valuable elements $(\mathrm{Cr}, \mathrm{Ni}, \mathrm{Mo}, \mathrm{V}$, etc.) are lost, too. The aim of this work is to study the feasibility for effective recycling of cold bonded by-products pellets (VOD/VD sludge, ladle furnace dust and scales generated in forging shop) in the electric arc furnace (EAF). Firstly, the raw materials were mixed together with fines of petroleum coke and cement. After pelletizing and curing period, the selfreducing pellets were melted in an induction furnace (lab-scale tests) in order to evaluate the recovery of the valuable elements from the steel mill by-products. The yields of $\mathrm{Cr}, \mathrm{Ni}$ and Mo varied from $84 \%$ to $99 \%$ in the lab-scale experiments. In the industrial test (EAF) by using self-reducing pellets of mill scale from forging shop ( $\mathrm{H} 13$ tool steel grade) the yield of $\mathrm{Cr}$, Mo and $\mathrm{V}$ varied from $51 \%-92 \%, 94 \%-99 \%$ and $27.5 \%-88 \%$, respectively.

Keywords: Recycling; Waste; Pellets.

SP, Brasil.

Engenheiro de Materiais, Gerente de Processos, Aciaria, Villares Metals S.A, Sumaré, SP, Brasil.

Engenheiro Metalurgista, Engenheiro de Processos, Aciaria, Villares Metals S.A, Sumaré, SP, Brasil.

Engenheiro Metalurgista, Engenheiro de Processos, Aciaria, Villares Metals S.A, Sumaré, SP, Brasil.

Engenheiro Metalurgista, Engenheiro de Processos, Aciaria, Villares Metals S.A, Sumaré, SP, Brasil.

Técnico em Metalurgia, Supervisor Operacional, Aciaria, Villares Metals S.A, Sumaré, Brasil, Brasil.

Engenheiro Civil, Gerente da Aciaria convencional, Villares Metals S.A, Sumaré, SP, Brasil.

Engenheiro Metalurgista, Diretor da Aciaria, Villares Metals S.A, Sumaré, SP, Brasil.

Engenheiro de Materiais, Dr., Diretor de Tecnologia, Villares Metals S.A, Sumaré, SP, Brasil.
\end{abstract}

\footnotetext{
* Contribuição técnica ao $45^{\circ}$ Seminário de Aciaria - Internacional, 25 a 28 de maio de 2014,
} Porto Alegre, RS, Brasil. 


\section{INTRODUÇÃO}

Basicamente dois aspectos motivam a procura por meios que solucionem os problemas advindos da geração de rejeitos em processos industriais. O primeiro de conotação puramente ambiental se fundamenta em pressões formais (legislação) ou informais (comunidade) sobre as empresas, para que estas enquadrem seus níveis de emissão a valores considerados toleráveis. O segundo, de caráter econômico, é uma decorrência do objetivo básico de qualquer empreendimento que é a minimização dos custos e melhoria dos resultados. Desta forma, a redução de emissões significa aumento de rendimento e redução na quantidade de matérias primas e insumos comprados por unidade de produto. Além disso, o seu reaproveitamento desde que em bases econômicas aceitáveis, reduz custo (uso interno) ou gera receita adicional (uso externo). Em resumo, a politica ambiental das empresas está fundamentada em base tanto de natureza social quanto ambiental.

Considerando os efluentes sólidos de um sistema qualquer onde, por exemplo, uma usina siderúrgica é um caso típico, são duas as situações possíveis:

- os efluentes sólidos são captados e descartados em aterros controlados;

- os efluentes sólidos, em sua maioria, são captados e reaproveitados interna ou externamente.

Normalmente estas duas situações coexistem dentro de um sistema, sendo que a intensidade de participação de cada uma varia de empresa para empresa e/ou de região para região.

Os padrões de identificação e descarte de resíduos industriais variam sensivelmente entre os países. Porém, praticamente todas as nações industrializadas ou em franco desenvolvimento tem revisto e tornado mais restritivas suas legislações ambientais, em especial no que concerne à geração, transporte, tratamento e descarte final dos resíduos industriais.

A reciclagem de resíduos industriais, tais como o da rota de produção do aço, representa um componente primordial do esforço para um desenvolvimento duradouro e sustentável. Além disso, ela guarda uma relação econômica importante com as práticas legais de descarte, cada vez mais restritivas. Muitas técnicas de tratamento de resíduos siderúrgicos têm sido desenvolvidas por todo o mundo, mas muito ainda deve ser feito quanto à recuperação de alguns deles, considerados nocivos e gerados em quantidades limitadas. Como consequência natural, ocorrerão investimentos crescentes em técnicas de tratamento de resíduos na própria usina, reciclagem por terceiros, obtenção de subprodutos ou em último caso, descarte em categoria menos onerosa.

Em usinas que produzem aços especiais, sendo um exemplo típico a Villares Metals SA, todas as lamas, carepas e pós gerados durante as etapas produtivas na aciaria, laminação, forjaria e acabamento, invariavelmente contém teores significativos de elementos de liga, tais como $\mathrm{Cr}, \mathrm{Ni}, \mathrm{Mo}, \mathrm{V}, \mathrm{W}$, etc. Pensando nesta possibilidade de recuperação destes elementos, pelotas autorredutoras de lama de VOD, poeira do forno panela e carepa de aço ferramenta $(\mathrm{H} 13)$ foram fundidas num forno de indução em caráter experimental, para avaliação dos rendimentos dos elementos de interesse ( $\mathrm{Cr}, \mathrm{Ni}, \mathrm{Mo}, \mathrm{V}$, etc.), servindo então de base para testes industriais.

\subsection{Geração de Poeiras na Produção do Aço}

Diversas pesquisas foram feitas com o objetivo de se entender o mecanismo gerador de pós durante a operação de conversores e fornos elétricos a arco [1-12].

* Contribuição técnica ao $45^{\circ}$ Seminário de Aciaria - Internacional, 25 a 28 de maio de 2014, Porto Alegre, RS, Brasil. 
Com este conhecimento sistemas de despoeiramento mais eficazes poderiam ser projetados, além também da minimização da quantidade de pós a ser formada. Em sua maioria os estudos consideram que os principais mecanismos de geração de poeiras são:

- ejeção de gotas do aço líquido e da escória;

- vaporização de elementos que apresentam alta pressão de vapor ( $\mathrm{Zn}, \mathrm{Pb}, \mathrm{Cd}$, etc.);

- arraste de materiais adicionados ao forno pelo sistema de despoeiramento.

O último mecanismo é importante em períodos quando os fundentes estão sendo adicionados ao forno, principalmente quando possuem certa parcela de material com granulação fina.

Na Villares Metals SA são geradas cerca de 500 t/ano de lama de VOD e 100 t/ano de poeira de forno panela. No entanto, a poeira de forno panela é descartada juntamente com a poeira de exaustão do FEA, apesar de serem materiais com características químicas e físicas distintas.

\subsection{Geração de Carepas na Forjaria}

Através de análises por microscopia ótica e eletrônica de varredura (MEV-EDS), Bagatini et al. [13] observaram que a carepa estudada por eles apresentava três zonas distintas com diferentes microestruturas e relações O/Fe, sugerindo que a camada mais externa fosse composta por hematita e magnetita e a mais interna por wustita.

A geração de carepas na forjaria da Villares Metals ocorre principalmente nas etapas de forja e tratamento térmico, num total de 3000 t/ano, sendo 250 t/ano somente do aço VH13. Em geral, a carepa produzida na forjaria está na forma de pequenos pedaços, escamas e placas com até $30 \mathrm{~mm}$ de espessura por $150 \mathrm{~mm}$ de comprimento, necessitando então de um processo de moagem, visando qualquer tipo de processo de aglomeração posterior.

\subsection{Processo de Autorredução}

O termo 'pelota autorredutora' descreve uma mistura de materiais pulverulentos (finos de minério de ferro, poeiras de forno elétrico a arco, conversor, VOD, etc) e um agente redutor também na forma pulverulenta (finos de coque, carvão mineral, vegetal e até mesmo FeSi em pó) aglomerados por alguma técnica de cura a frio. Os aglomerantes mais usuais são o cimento Portland, escória de alto-forno, cal e aglomerantes orgânicos. Após o processo de cura, que pode levar algumas semanas, a pelota deve ter resistência suficiente ao manuseio e também nas altas temperaturas de processamento, ou seja, não sofrer crepitação e inchamento catastrófico. De fato, a redução de diversos óxidos (Fe, Mo, Ni, etc.) por carbono sólido, quando aglomerados conjuntamente na forma de pelotas ou briquetes, apresenta vantagens tecnológicas e cinéticas importantes, devido à granulometria fina, contato íntimo entre as partículas, geração interna do agente redutor gasoso e também pelas pequenas distâncias de difusão gasosa. Essas características levaram ao desenvolvimento de pesquisas com o objetivo de empregar este tipo de aglomerado em equipamentos tais como fornos cubilô, fornos tipo cuba, fornos rotativos e de soleira rotativa [14].

Muito tem sido estudado sobre o mecanismo de redução dos óxidos de ferro por redutor sólido (finos de carvão mineral, vegetal, coque e grafite), envolvendo

* Contribuição técnica ao $45^{\circ}$ Seminário de Aciaria - Internacional, 25 a 28 de maio de 2014, 
reações químicas e transferência de massa entre eles [15]. De fato, há um consenso que a reação global de redução ocorre por intermediários gasosos, produto das seguintes reações que ocorrem em paralelo:

$$
\begin{aligned}
& \mathrm{Fe}_{2} \mathrm{O}_{3(\mathrm{~s})}+1 / 3 \mathrm{CO}_{(\mathrm{g})}=2 / 3 \mathrm{Fe}_{3} \mathrm{O}_{4(\mathrm{~s})}+1 / 3 \mathrm{CO}_{2(\mathrm{~g})} \\
& 2 / 3 \mathrm{Fe}_{3} \mathrm{O}_{4(\mathrm{~s})}+2 / 3 \mathrm{CO}_{(\mathrm{g})}=2 \mathrm{FeO}_{(\mathrm{s})}+2 / 3 \mathrm{CO}_{2(\mathrm{~g})} \\
& 2 \mathrm{FeO}_{(\mathrm{g})}+2 \mathrm{CO}_{(\mathrm{g})}=2 \mathrm{Fe}_{(\mathrm{s})}+2 \mathrm{CO}_{2(\mathrm{~g})} \\
& \mathrm{CO}_{2(\mathrm{~g})}+\mathrm{C}_{(\mathrm{s})}=2 \mathrm{CO}_{(\mathrm{g})}
\end{aligned}
$$

A estequiometria da reação global pode ser representada por:

$\mathrm{Fe}_{2} \mathrm{O}_{3(\mathrm{~s})}+p \mathrm{C}_{(\mathrm{s})}=2 \mathrm{Fe}_{(\mathrm{s})}+u \mathrm{CO}_{(\mathrm{g})}+v \mathrm{CO}_{2(\mathrm{~g})}$

Como mostrado na equação (5) e de acordo com Mourão [15], não há uma estequiometria definida para a reação global e os coeficientes $p, u$ e $v$ assumem valores que dependem das velocidades relativas das reações parciais, isto é, da reação de redução do óxido por $\mathrm{CO}_{(\mathrm{g})}$ e da reação de Boudouard.

\section{MATERIAIS E MÉTODOS}

\subsection{Matérias-Primas}

As matérias-primas utilizadas neste trabalho foram: (1) lama de VOD/VD; (2) poeira de forno panela (FP); (3) carepa de forjaria (aço H13); (4) finos de coque de petróleo; (5) $\mathrm{CaF}_{2}$; (6) Alumínio metálico (99,98\%); (7) Ferro eletrolítico (99,98\%); e (8) cimento Portland.

\subsection{Resíduos}

Todas as amostras foram secas por 24 horas a $130^{\circ} \mathrm{C}$. A amostra de carepa, como se apresentava na forma de placas e escamas, passou por uma etapa de moagem, onde se aproveitou a fração -200\# visando a produção das pelotas. Após isto, todos os resíduos (lama de VOD/VD, poeira de forno panela e carepa de forjaria (aço H13)) foram caracterizados no que tange a sua composição química e granulometria.

As Tabelas 1 e 2 mostram as composições químicas e as distribuições granulométricas dos resíduos (lama de VOD e poeira de forno panela) utilizados neste trabalho, respectivamente. Cabe lembrar que no caso da carepa utilizou-se somente a fração $-200 \#$ na produção das pelotas autorredutoras.

* Contribuição técnica ao 45 Seminário de Aciaria - Internacional, 25 a 28 de maio de 2014, 
Tabela 1. Composição química dos resíduos

\begin{tabular}{|cccc|}
\hline Elementos & Lama VOD (\%) & Poeira forno panela (\%) & Carepa (\%) \\
\cline { 4 - 4 } C & 0,35 & 0,13 & 0,004 \\
$\mathrm{Al}$ & 0,06 & 0,012 & 0,003 \\
$\mathrm{Cr}$ & 0,14 & 0,032 & 0,11 \\
$\mathrm{Mn}$ & 5,2 & 1,0 & 1,5 \\
$\mathrm{Mo}$ & 5,9 & 0,27 & 0,34 \\
$\mathrm{Ni}$ & 0,13 & 0,4 & 0,27 \\
$\mathrm{~V}$ & 1,4 & 0,33 & 0,05 \\
$\mathrm{Si}$ & 0,005 & 0,10 & 0,22 \\
$\mathrm{Ca}$ & 0,6 & 0,15 & 0,32 \\
$\mathrm{Mg}$ & 0,6 & 0,30 & na \\
$\mathrm{Cu}$ & 1,1 & 0,02 & na \\
$\mathrm{Pb}$ & 0,6 & 0,036 & 0,04 \\
$\mathrm{Sn}$ & 0,17 & 0,003 & na \\
$\mathrm{Zn}$ & 0,02 & 0,003 & na \\
$\mathrm{P}$ & 0,47 & 0,005 & na \\
$\mathrm{Fe}$ & 0,012 & 0,004 & 0,007 \\
\hline
\end{tabular}

na $=$ não analisado

Tabela 2. Distribuição granulométrica das partículas da lama de VOD/VD e poeira de forno panela

\begin{tabular}{|c|c|c|c|}
\hline \multicolumn{2}{|c|}{ Lama VOD } & \multicolumn{2}{|c|}{ Poeira de FP } \\
\hline$\mu \mathrm{m}$ & $\%$ & $\mu \mathrm{m}$ & $\%$ \\
\hline$<95.0$ & 100 & $<97,2$ & 79,4 \\
\hline$<49,7$ & 100 & $<54,0$ & 68,8 \\
\hline$<25,2$ & 97,3 & $<35,2$ & 50,0 \\
\hline$<15,0$ & 92,8 & $<20,1$ & 39,7 \\
\hline$<10,2$ & 87,5 & $<10,2$ & 25,6 \\
\hline$<4,0$ & 50,0 & $<5,0$ & 16,8 \\
\hline$<1,0$ & 9,2 & $<1,2$ & 3,1 \\
\hline
\end{tabular}

\subsection{Agente Redutor}

Como agente redutor utilizou-se coque de petróleo (finos), apresentando $98,7 \%$ de carbono fixo, $0,64 \%$ de matéria volátil, $0,7 \%$ de cinzas e $0,23 \%$ de enxofre.

\subsection{Aglomerante}

Como aglomerante utilizou-se cimento Portland de alta resistência inicial (ARI). A Tabela 3 mostra a sua composição química.

Tabela 3. Composição química do aglomerante

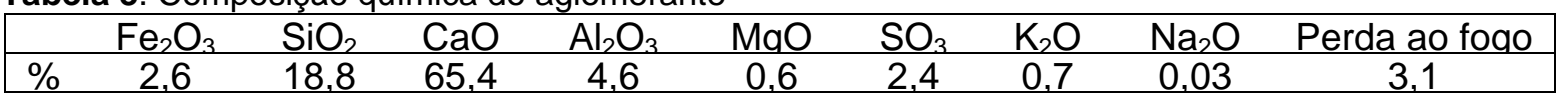

\subsection{Fundente}

Para fluidificar a escória formada durante a fusão das pelotas autorredutoras adicionou-se $\mathrm{CaF}_{2}(95,6 \%)$, tendo como principais impurezas $\mathrm{SiO}_{2}(2,1 \%), \mathrm{P}(0,8 \%)$ e $S(0,1 \%)$.

\subsection{Pelotização}

Nos experimentos laboratoriais as pelotas autorredutoras foram produzidas manualmente e em todos os casos utilizou-se $10 \%$ a mais de agente redutor além

* Contribuição técnica ao $45^{\circ}$ Seminário de Aciaria - Internacional, 25 a 28 de maio de 2014, Porto Alegre, RS, Brasil. 
do valor estequiométrico. Porém, no teste industrial, as pelotas autorredutoras de carepa da forjaria foram produzidas em disco pelotizador $\left(\varnothing_{\text {prato }}=2000 \mathrm{~mm}\right.$ e lateral de $300 \mathrm{~mm}$ ). As misturas utilizadas possuem uma combinação de resíduo, coque e cimento, na qual a quantidade de coque e cimento foram adicionadas para promover, respectivamente, a redução necessária e a resistência mecânica. Após a pelotização as pelotas passaram por um período de cura de pelo menos 1 mês, visando um ganho em resistência que fosse suficiente para o seu manuseio nas etapas posteriores. Após o período de cura e antes da etapa de fusão as pelotas foram secas por $24 \mathrm{~h}$ a $130^{\circ} \mathrm{C}$.

\subsection{Fusão das Pelotas Autorredutoras}

Nos testes laboratoriais as pelotas autorredutoras foram fundidas num forno de indução de média frequência. Para a fusão utilizou-se um cadinho de grafite de $250 \mathrm{~cm}^{3}$ de capacidade. Como não se formaria um campo indutivo para um volume tão pequeno para um forno com capacidade para $100 \mathrm{~kg}$, colocou-se um cadinho de grafite (eletrodo de forno panela usinado) dentro do forno de indução para servir como "susceptor". Dentro deste cadinho de grafite colocou-se então o cadinho de trabalho contendo previamente a massa de ferro eletrolítico ( $\mathrm{Fe}=99,99 \%$ ) que variou de 100 a $200 \mathrm{~g}$. Após a fusão do ferro eletrolítico (pé de banho), as pelotas autorredutoras foram paulatinamente adicionadas ao banho metálico. Do mesmo modo, paulatinamente, visando fluidificar a escória formada e também reduzi-la, fluorita e alumínio metálico foram adicionados ao banho. Nove fusões foram efetuadas, três para cada tipo de pelota (lama de VOD/VD, poeira de FP e carepa de forjaria). A temperatura do banho metálico durante a fusão no forno de indução variou de $1550-1580^{\circ} \mathrm{C}$.

Para os testes industriais no forno elétrico a arco (FEA) foram produzidas $5,5 \mathrm{t}$ de pelotas autorredutoras de carepa de forjaria (aço $\mathrm{H} 13$ ). Em geral, as pelotas foram carregadas na sola do FEA, isto é, sem a presença de aço líquido no interior do forno. Em algumas vezes as pelotas foram adicionadas diretamente no aço líquido, após a fusão da carga do primeiro cestão, mas a evolução de chamas é significativa. O carregamento juntamente com o primeiro cestão também foi outra opção explorada. A adição variou de 1 a 2,5 t e envolveu diferentes tipos de aço ferramenta, desfosforados ou não.

De posse da composição e das massas de entrada e saída dos experimentos de fusão, foi possível realizar o balanço de massa e assim calcular o rendimento dos elementos de liga contidos em cada resíduo.

\section{APRESENTAÇÃO E DISCUSSÃO DOS RESULTADOS}

\subsection{Fusão das Pelotas Autorredutoras de Lama de VOD/VD}

Dentre os resíduos estudados (lama de VOD/VD) é o que apresenta maiores teores de cal, sílica, alumina, etc., gerando assim mais escória. A Tabela 4 mostra a composição química (média dos três testes) do produto metálico da fusão das pelotas autorredutoras de lama de VOD. Como o cadinho era de grafite, o banho incorporou carbono. A Figura 1 mostra os rendimentos dos elementos de liga obtidos nos testes de fusão das pelotas de lama de VOD no forno de indução. Os rendimentos variaram de 95 a 97\% para o Cr, 87 a 92\% para o Ni e 88 a 93\% para o $\mathrm{Mn}$.

* Contribuição técnica ao 45 Seminário de Aciaria - Internacional, 25 a 28 de maio de 2014, 


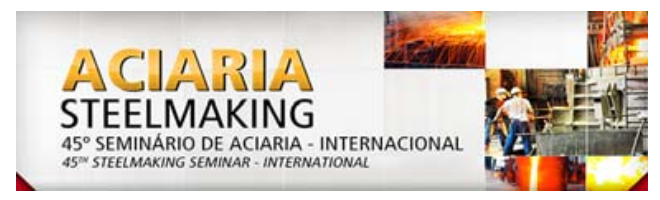

Tabela 4.Composição química (média) dos produtos metálicos das fusões das pelotas de lama de VOD

\begin{tabular}{|llllllllll|}
\hline$\%$ & $\mathrm{C}$ & $\mathrm{P}$ & $\mathrm{Pb}$ & $\mathrm{S}$ & $\mathrm{Sn}$ & $\mathrm{Cu}$ & $\mathrm{Cr}$ & $\mathrm{Ni}$ & $\mathrm{Mn}$ \\
\hline & 4,1 & 0,0093 & 0,0031 & 0,007 & 0,017 & 0,6 & 9,9 & 1,6 & 8,1 \\
\hline
\end{tabular}

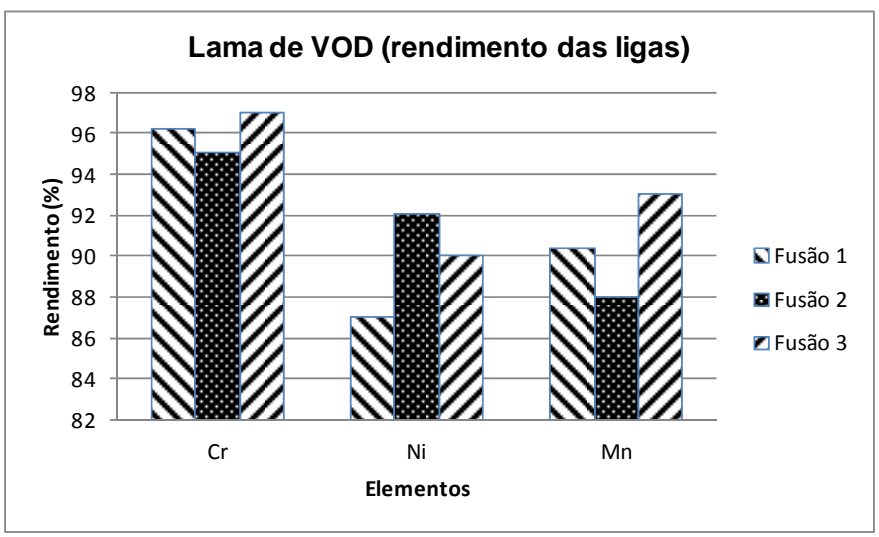

Figura 1. Rendimento dos elementos de liga obtidos na fusão das pelotas de lama de VOD.

Um dos problemas na reciclagem da lama de VD/VOD ao FEA poderia ser a presença de alguns elementos deletérios às propriedades do aço, tais como $\mathrm{Pb} e$ Sn. No produto metálico oriundo das fusões das pelotas de lama de VOD/VD, os teores variaram de 28 a 36 ppm para o $\mathrm{Pb}$ e 160 a 185 ppm para o Sn. De fato, para não desvirtuar o propósito principal do FEA, que é fundamentalmente uma unidade de fusão, não mais que uma tonelada de pelotas seria adicionada ao FEA. De modo que as $500 \mathrm{t}$ de lama geradas por ano seriam consumidas num universo de aproximadamente 2000 corridas/ano.

\subsection{Fusão das Pelotas Autorredutoras de Poeira de Forno Panela}

A Tabela 5 mostra a composição química (média dos três testes) do produto metálico da fusão das pelotas de poeira de forno panela. Cromo, níquel e molibdênio na realidade advêm de uma série de aços produzidos neste forno panela, compreendendo aços para construção mecânica, ferramenta e rápido. Pequenas variações de composição podem ocorrer no produto metálico da fusão da poeira de forno panela, motivado pela gama de aços produzidos, mas nada que fuja destes três elementos.

A Figura 2 mostra os rendimentos dos elementos de liga obtidos nos testes de fusão das pelotas. Para $\mathrm{Cr}$, Ni e Mo os rendimentos variaram de 73,4 a 77\%, 98,9 a 99,8\% e 98,1 a $98,7 \%$, respectivamente. É importante mencionar a presença de muitas partículas metálicas na poeira de forno panela, como pode ser notado na Figura 3 e Tabela 6. Isto evidencia o mecanismo de formação do material, que se dá principalmente pela ejeção de partículas do banho metálico. Trata-se realmente de um resíduo que pode e deve ser reaproveitado devido ao seu elevado teor metálico e poucos contaminantes. Do mesmo modo que nas fusões das pelotas de lama de VOD, carbono foi incorporado pelo banho metálico (cadinho de grafite).

Tabela 5. Composição química (média) dos produtos metálicos das fusões das pelotas de poeira de forno panela

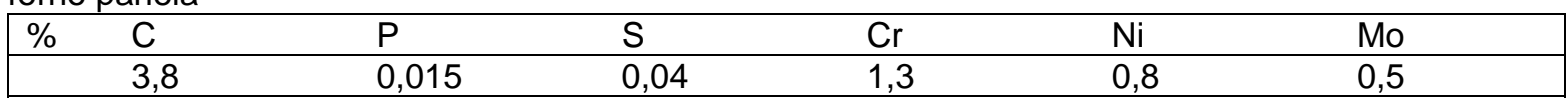

* Contribuição técnica ao 450 Seminário de Aciaria - Internacional, 25 a 28 de maio de 2014, 

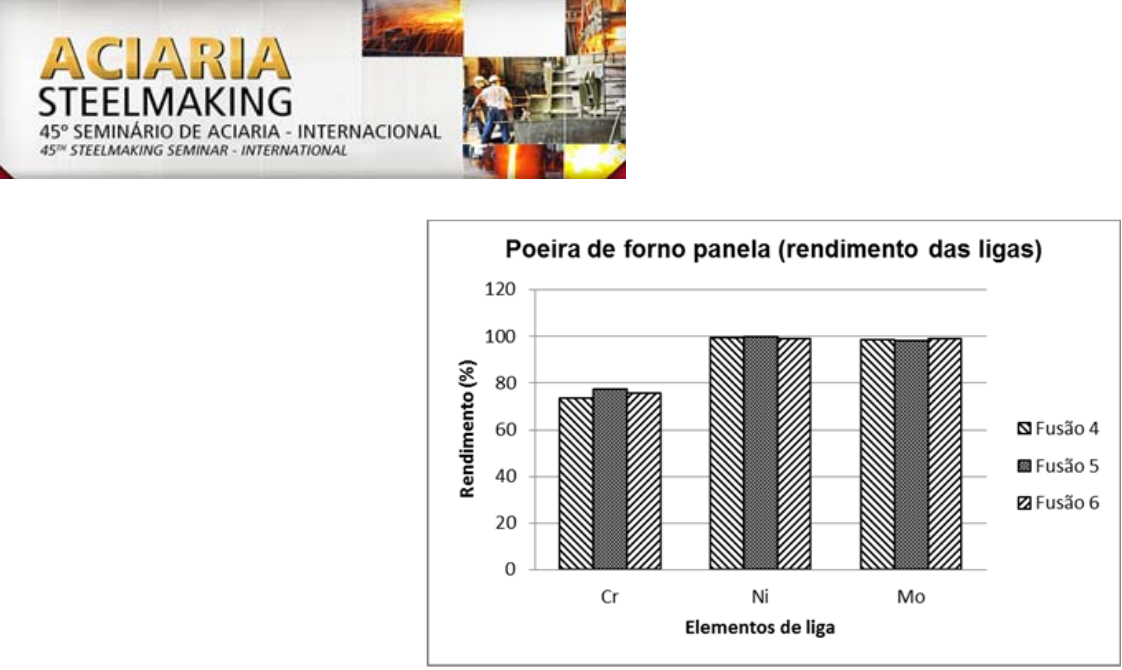

Figura 2. Rendimento dos elementos de liga obtidos na fusão das pelotas de poeira de forno panela.

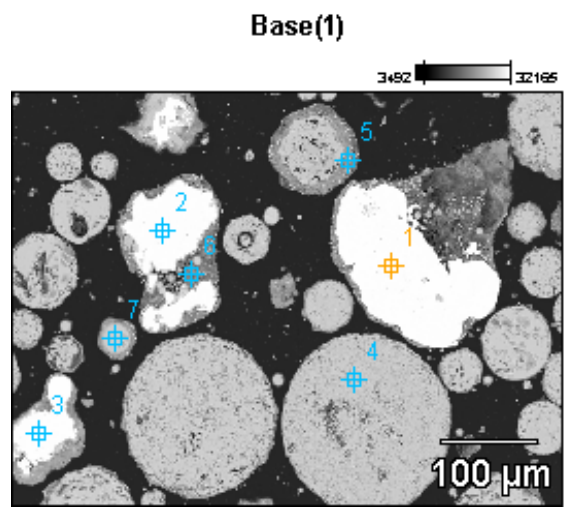

Figura. 3 Seção polida das partículas de poeira de forno panela. Microscopia eletrônica de varredura (eletrons retroespalhados).

Tabela 6. Resultados da microanálise por EDS dos pontos mostrados na Figura 3

\begin{tabular}{|cccccccccc|}
\hline$\%$ & $\mathrm{C}$ & & \multicolumn{10}{c|}{$\mathrm{Fe}$} & & \\
\hline 1 & 3 & --- & & & --- & --- & 97,0 & --- & --- \\
2 & 2,9 & --- & --- & -- & -- & -- & 96,9 & --- & -- \\
3 & 2,9 & --- & -- & -- & -- & -- & 96,9 & -- & - \\
$\%$ & --- & $\mathrm{Al}_{2} \mathrm{O}_{3}$ & $\mathrm{SiO}_{2}$ & $\mathrm{~V}_{2} \mathrm{O}_{5}$ & $\mathrm{Cr}_{2} \mathrm{O}_{3}$ & $\mathrm{MnO}$ & $\mathrm{Fe}_{2} \mathrm{O}_{3}$ & $\mathrm{NiO}$ & $\mathrm{MoO}_{3}$ \\
4 & --- & --- & 4,2 & 3,5 & 8,7 & 1,4 & 82,2 & --- & --- \\
5 & --- & 0,7 & 1,5 & --- & 11,7 & 1,2 & 71,8 & 7,5 & 5,6 \\
6 & --- & --- & 1,6 & --- & 6,7 & --- & 83,5 & 2,7 & 5,5 \\
7 & --- & --- & 0,6 & --- & 2,1 & --- & 95,7 & --- & 1,6 \\
\hline
\end{tabular}

\subsection{Fusão das Pelotas Autorredutoras de Carepa Gerada na Forjaria (Aço H13)}

A Tabela 7 mostra a composição química (média dos três testes) do produto metálico da fusão das pelotas de carepa de forjaria (aço H13) no forno de indução. Notar que houve a incorporação ao banho metálico dos principais elementos de liga do aço ferramenta H13. Houve também incorporação de C (cadinho de grafite).

Tabela 7. Composição química do produto metálico da fusão das pelotas de carepa de forjaria

\begin{tabular}{|lllllll|}
\hline$\%$ & $\mathrm{C}$ & $\mathrm{P}$ & $\mathrm{S}$ & $\mathrm{Cr}$ & $\mathrm{V}$ & $\mathrm{Mo}$ \\
\hline & 4,5 & 0,017 & 0,02 & 3,9 & 0,5 & 1,2 \\
\hline
\end{tabular}

A Figura 4 mostra os rendimentos dos elementos de liga obtidos nos testes de fusão das pelotas autorredutoras de carepa. Para $\mathrm{Cr}, \mathrm{V}$ e Mo os rendimentos variaram de 84 a $87 \%, 85$ a $90 \%$ e 90 a 93,5\%, respectivamente. Tal como nos testes com a poeira de forno panela, o teor de $\mathrm{P}$ pode até ser considerado elevado em se tratando de aços que não serão desfosforados. Cabe então considerar tal "pick up" de fósforo

* Contribuição técnica ao $45^{\circ}$ Seminário de Aciaria - Internacional, 25 a 28 de maio de 2014, Porto Alegre, RS, Brasil. 


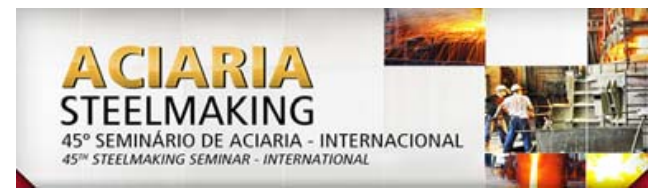

oriundo da adição de pelotas, não mais que $2,5 \mathrm{t}$, na composição da carga total do FEA e adições no forno panela, de modo que o teor de fósforo não ultrapasse o especificado em norma.

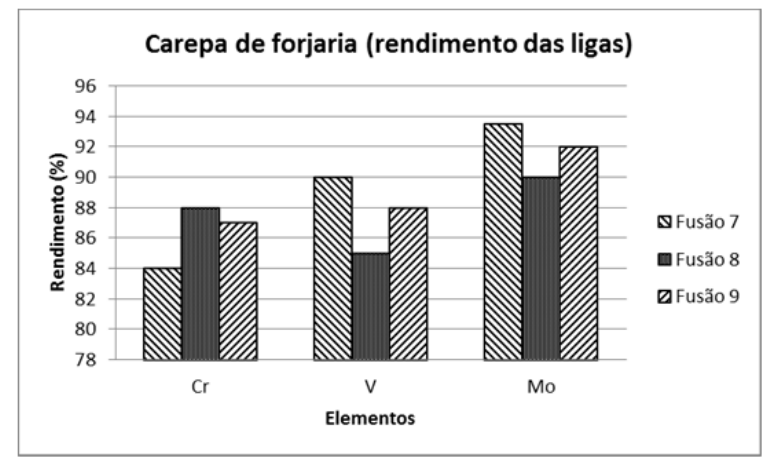

Figura 4. Rendimento dos elementos de liga dos testes de fusão das pelotas de carepa.

A Figura 5 mostra os resultados de rendimento dos elementos de liga ( $\mathrm{Cr}$, Mo e V) quando da adição das pelotas de carepa de forjaria ao FEA. Independente da corrida, desfosforada ou não, o rendimento do Mo foi excelente. Este resultado está condizente com testes realizados com pelotas autorredutoras de $\mathrm{MoO}_{3}$ no FEA da Villares Metals S.A. e também com o estudo de SONG et al. [16]. Por outro lado, o rendimento dos elementos $\mathrm{Cr}$ e $\mathrm{V}$ foram os piores na corrida desfosforada, onde a condição reinante no banho era mais oxidante, fato este que não ocorre quando se utiliza sucata retorno dos aços em questão (VC131 e VD2), situação na qual as condições são menos oxidantes no banho metálico.

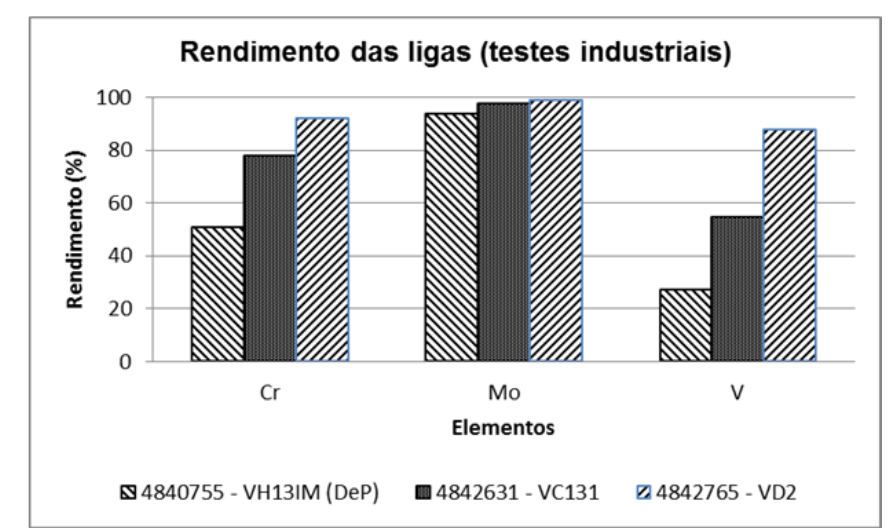

Figura 5. Rendimento dos elementos de liga dos testes industriais no FEA (carepa de forjaria).

\section{CONCLUSÕES}

Nos testes laboratoriais envolvendo a adição das pelotas autorredutoras de lama de VD/VOD, poeira de forno panela e carepa a um banho metálico (ferro eletrolítico), o rendimento dos elementos $\mathrm{Cr}, \mathrm{Ni}$, Mo e Mn sempre foi superior a $70 \%$.

Nos testes industriais envolvendo somente as pelotas de carepa de forjaria (H13), os menores rendimentos, principalmente de cromo e vanádio (foram obtidos na corrida que foi desfosforada, situação na qual onde não se utilizou sucata do próprio aço a ser produzido.

A aglomeração de diversos materiais particulados (resíduos) gerados durante a produção do aço, na forma de pelotas e/ou briquetes autorredutores, é uma maneira eficaz de se recuperar diversos elementos de liga que antes seriam descartados.

* Contribuição técnica ao $45^{\circ}$ Seminário de Aciaria - Internacional, 25 a 28 de maio de 2014, 


\section{REFERÊNCIAS}

1 Turkdogan ET, Leake LE. Preliminary studies on the evolution of fumes from iron at high temperatures. Journal of the Iron and Steel Institute. 1959;192(6):162-70.

2 Turkdogan ET, Grieveson P, Darken LS. The formation of iron oxide fume. Journal of Metals. 1962;14(7):521-26.

3 Morris JP, Riott JP, lilig EG. A new look at cause of fuming. Journal of Metals. 1966;18(7):803-10.

4 Ellis AF, Glover J. Mechanism of fume formation in oxygen steelmaking. Journal of the Iron and Steel Institute. 1971;210(9):593-99.

5 Baker LA, Ward RG. Reaction an iron carbon droplet during free fall through oxygen. Journal of the Iron and Steel Institute. 1967;205(7):714-17.

6 See JB, Warner NA. Reactions of iron alloy drops in free fall through oxidinzing gases. Journal of the Iron and Steel Institute. 1973;211(1):44-52.

7 Roddis PG. Mechanism of descarburization of iron-carbon alloy drops falling through an oxidizing gas. Journal of the Iron and Steel Institute. 1973;212(1):53-8.

$8 \mathrm{Li}$ CL, Tsai MS. Mechanism of spinel ferrite dust formation in electric arc furnace steelmaking. ISIJ International. 1993;29(4):284-90.

9 Delhaes C, Hauck A, Neuschutz D. Mechanism dust generation in a stainless steelmaking converter. Steel Research. 1993;64(1):22-7.

10 Tsujino R, Hirai M, Ohno T, Ishiwata N, Inoshita, T. Mechanism of dust generation in a converter with minimum slag. ISIJ International. 1989;29(4):291-99.

11 Nedar L. Dust formation in a BOF converter. Steel Research. 1996;67(8):320-27.

12 Mantovani MC, Takano C, Buchler PM. EAF and secondary dust characterisation. Ironmaking \& Steelmaking. 2004;31(4):325-32.

13 Bagatini MC, Zymla V, Osório E, Vilela ACF. Characterization and reduction behavior of mill scale. ISIJ International. 2011;51(7):1072-79.

14 Mantovani MC, Takano C, Buchler PM. Ironmaking \& Steelmaking. 2002;29(4):257-65.

15 Mourão MB. Análise do processo de redução de minério de ferro por carbono na forma de pelotas autorredutoras [tese de doutorado]. São Paulo: Escola Politécnica, Universidade de São Paulo; 1988. p.191.

16 Song S-Q, Xue Z-L, Yu, Y, Liu R-N, Wang G-L. ISIJ International. 2013;53(7):1138-42.

* Contribuição técnica ao 45 Seminário de Aciaria - Internacional, 25 a 28 de maio de 2014, 